\title{
December Issue of Stem Cell Reviews and Reports Highlights Future Directions in the Mobilization of Hematopoietic Stem Cells and Presents Clinical Results of the First CAR-T Cell Treatment for Systemic Lupus Erythromatosus
}

\author{
Mariusz Z. Ratajczak ${ }^{1}$ \\ Published online: 2 November 2021 \\ ( $)$ The Author(s), under exclusive licence to Springer Science+Business Media, LLC, part of Springer Nature 2021
}

This last in the current year December issue of Stem Cell Reviews and Reports includes several interesting papers on the role of stem cells in tissue and organ regeneration. Taking an opportunity, we highlight two inspiring articles which desire the special attention of our readership. In the first, Dr. Johnatan Hoggatt from the Center for Cancer Research and Transplantation Sciences at Massachusetts General Hospital, Boston, discusses currently available approaches for mobilizing hematopoietic stem cells (HSCs), addresses cellular heterogeneity of mobilized cell products and proposes new methods for HSCs mobilization and transplant conditioning.

Stem cell mobilization is still a significant clinical problem. As Dr. Hoggatt stated, there are in the clinic very often cases of poor mobilizers under currently available mobilization protocols, including patients suffering from lymphoid malignancies, and even sometimes it is also difficult to harvest enough cells from normal healthy donors. To support this, as reported, up to $40 \%$ of patients with multiple myeloma (MM) do not mobilize after routine administration of pro-mobilizing cytokine granulocyte-colony factor (Filgrastim) enough HSCs for auto-transplantation and require more intensive protocols by addition of CXCR4 receptor antagonist - AMD3100 (Plerixafor).

Ten years ago, I had an opportunity to serve as quest editor for the special issue on stem cell mobilization in Leukemia. As an introduction for this series, I proposed a provocative title, "Spotlight Series on Stem Cell Mobilization: Many Hands on the Ball, but Who is the Quarterback?". Today, ten years later, we still do not understand completely all sometimes-redundant mechanisms involved in the release of HSCs from bone marrow (BM) into peripheral blood

Mariusz Z. Ratajczak

mariusz.ratajczak@louisville.edu

1 University of Louisville, Louisville, KY, USA
(PB). Overall, the mobilization process is directed by $i$ ) a decrease in SDF-1-CXCR4 and VLA-4-VCAM-1 interactions in $\mathrm{BM}, \mathrm{ii}$ ) reversal of the trans-endothelial chemotactic gradient between the BM and plasma, iii) activation of the complement and coagulation cascades and $i v$ ) release of neurotransmitters from the synapses of the nerves that innervate the BM microenvironment.

In his current spotlight, Dr. Hoggatt reviews recent clinical developments and new mobilization protocols employing to standard pro-mobilizing agents' addition of non-steroid anti-inflammatory drug meloxicam or small chemokine: growth-regulated protein beta (Gro- $\beta$ ). Of note in the same December issue, it is also published an original clinical trial paper from Dr. Pelus's group that reports the application of meloxicam with Filgrastim in 25 patients with MM. Furthermore, Dr. Hoggatt in his spotlight paper also presents a perspective on how to improve potentially the mobilization process by i) permeabilizing the BM-PB barrier in the presence of neuropeptide $\mathrm{Y}$, ii) application of sildenafil citrate or medicinal marijuana compounds, and finally iii) by activation of nociceptive nerves in BM. What is important Dr. Hoggatt, in his final remarks, stated that he does not agree with some "opinion-makers" that there is no longer a reason to study HSC mobilization. In contrast, he wrote that this next decade might ultimately reflect a golden era of HSCs mobilization research and provides several arguments to support this claim in his excellent spotlight.

In another exciting paper published in the December issue of Stem Cell Reviews and Reports, Dr. Yupo Ma group iCell Gene Therapeutics, Stony Brook, NY, USA and his collaborators from University Shenzhen in Beijing, PR. China present encouraging data on the clinical application of a novel CAR-T cells compound-BCMA-CD19 in the treatment of systemic lupus erythromatosus (SLE). This new original modification of CAR-T cells has been developed to target at the same time CD19 on B cells and CD269 (BCMA) on 
plasma cells. This dual-action of the new CAR-T cell compound seems to be very effective for the treatment of SLE and other autoimmunity disorders. Dr. Ma concludes that it is possible to reset the antibody-producing "root" B cells and plasma cells by employing this strategy, what leads to favorable clinical outcomes. What is also worth to mention, Dr. Ma's group published in the past two years in our journal few excellent papers on the application of CAR-T strategies in the treatment of hematopoietic malignancies, and we encourage to follow his pioneering work in this area.

Finally, taking an opportunity, our editorial team also would like to congratulate all those authors whose excellent papers have been accepted for publication in this December issue and express special thanks to our dedicated reviewers. They help us to establish Stem Cell Reviews and Reports as one of the most influential journals in the area of stem cell research.

Publisher's Note Springer Nature remains neutral with regard to jurisdictional claims in published maps and institutional affiliations. 